\title{
Relationship between dietary folate intake and plasma monocyte chemoattractant protein-1 and interleukin-8 in heart failure patients
}

\author{
Hye Kyung Chung, ${ }^{1,2}$ Oh Yoen Kim, ${ }^{3}$ Hyeran Lee, ${ }^{1}$ Hyun Joo Do, ${ }^{1}$ Young Soon Kim, ${ }^{1}$ Jaewon Oh, ${ }^{4}$ \\ Seok-Min Kang ${ }^{4,5}$ and Min-Jeong Shin ${ }^{1, *}$ \\ 1 Institute of Health Science and Department of Food and Nutrition, College of Health Science, Korea University, Seoul 136-703, Republic of Korea \\ ${ }^{2}$ Department of Nutrition Services, Gangnam Severance Hospital, Yonsei University College of Medicine, Seoul 135-720, Republic of Korea \\ ${ }^{3}$ Yonsei University Research Institute of Science for Aging, Yonsei University, Seoul 120-749, Republic of Korea \\ ${ }^{4}$ Cardiology Division, Yonsei Cardiovascular Center and Cardiovascular Research Institute, Yonsei University College of Medicine, \\ Seoul 120-752, Republic of Korea \\ ${ }^{5}$ Brain Korea 21 Project for Medical Science, Yonsei University College of Medicine, Seoul 120-752, Republic of Korea
}

(Received 6 October, 2010; Accepted 27 November, 2010; Published online 30 June, 2011)

\begin{abstract}
This study aimed to examine the association of dietary vitamin intakes with plasma pro-inflammatory cytokine levels in Korean heart failure patients. Stable outpatients with heart failure were recruited and finally 91 patients were included. Dietary intakes were estimated by a developed semi-quantitative food frequency questionnaire. The simultaneous measurement of 17 cytokines was performed along with analysis of plasma C-reactive protein. Plasma C-reactive protein levels significantly correlated with dietary intakes of vitamin $C(r=-0.30, p<0.005), \beta$-carotene $(r=-0.23$, $p<0.05)$, and folate $(r=-0.31, p<0.005)$. However, these associations were no longer significant after adjusting for traditional risk factors for heart failure. On the other hand, plasma levels of monocyte chemoattractant protein-1 significantly correlated with dietary folate intake $(r=-0.31, p<0.001)$, and plasma interleukin-8 levels significantly correlated with dietary intakes of vitamin $C$ $(r=-0.38, p<0.001), \beta$-carotene $(r=-0.42, p<0.001)$, and folate $(r=-0.38, p<0.001)$ after the adjustment. Dietary folate intake was found as a primary influencing factor on plasma levels of monocyte chemoattractant protein-1 $\left(p<0.005, R^{2}=0.20\right)$ and interleukin-8 $\left(p<0.001, R^{2}=0.32\right)$ through a stepwise multiple linear regression analysis. Dietary folate intake was significantly associated with plasma levels of monocyte chemoattractant protein-1 and interleukin-8 which indicates dietary folate may have a potentially beneficial role in the prevention and treatment of heart failure.
\end{abstract}

Key Words: dietary folate, inflammation, heart failure, interleukin-8, MCP-1

A ccumulating data have suggested that various cytokines play important roles in the development and the progression of heart failure (HF). ${ }^{(1)}$ Monocyte activation in the myocardium under conditions of myocardial injury is postulated to be associated with the increased production of pro-inflammatory cytokines in HF, which possibly affects cardiac remodeling and peripheral disturbance. ${ }^{(2-5)}$ It has been consistently reported that circulating levels of tumor necrosis factor alpha (TNF- $\alpha)$ and interleukin (IL) -6 are elevated in patients with $\mathrm{HF}^{\left({ }^{(6)}\right.}$ and a direct relationship between the levels of these cytokines and deteriorating functional classes of HF. ${ }^{(7-8)}$

On the other hand, antioxidant vitamins are reported to modulate pro-inflammatory pathways in numerous diseases. ${ }^{(9-11)}$ Antioxidant vitamins may protect against oxidant-mediated inflammation and tissue damage by scavenging free radicals and inhibiting oxidant-sensitive transcription factors. ${ }^{(7)}$ Plasma levels of antioxidant vitamins such as $\beta$-carotene, $\alpha$-carotene and lycopene are known to reflect dietary intake of these vitamins. ${ }^{(12-15)}$ Animal and in vitro studies also showed that dietary factors such as antioxidants and fatty acids may modulate the expression of pro-inflammatory cytokines. ${ }^{(8,16)}$ Considering that micronutrient deficiencies are very common in HF patients and potentially contribute to the cause of $\mathrm{HF},{ }^{(8)}$ the maintenance of adequate antioxidant status may be useful in attenuating the cellular injury and inflammatory dysfunction observed in HF. In line with this evidence, a recent study suggested that long-term micronutrient supplementation in elderly HF patients can improve left ventricular function and quality of life. ${ }^{(17)}$

Taken together, certain dietary vitamins which have antioxidant properties may be associated with levels of pro-inflammatory cytokines, which can be an important factor for the pathogenesis and the progression of HF. Therefore, the present study examined the associations of antioxidant vitamin intakes from usual diet with plasma levels of pro-inflammatory cytokines along with $\mathrm{C}$ reactive protein (CRP) as a surrogate inflammatory marker in patients with $\mathrm{HF}$.

\section{Materials and Methods}

Study subjects. Ninety-one eligible consecutive ambulatory HF patients from an HF outpatient clinic at Yonsei Cardiovascular Center participated in this cross-sectional study (Mar 2008-Oct 2008). The inclusion criteria were: (1) diagnosis of systolic HF with a left ventricular ejection fraction (LVEF) $\leq 50 \%$, (2) $\leq 80$ years of age, and (3) stable HF through medication for at least 1 month prior to inclusion. We excluded patients with (1) a diagnosis of HF with preserved ejection fraction $(>50 \%),(2)$ a diagnosis of acute myocardial infarction 3 months prior to inclusion, (3) severe cognitive impairment and (4) antioxidant vitamin supplementation. The patients were given written informed consent, and the Institutional Review Board at Yonsei University Medical Center approved the study protocol. Medical history, including diagnosis, underlying disease, etiology of HF, and drug use, was obtained from the hospital database.

Anthropometric parameters and blood collection. Body weight and height were measured unclothed and without shoes in

*To whom correspondence should be addressed. E-mail: mjshin@korea.ac.kr 
Table 1. Baseline characteristics of the total subjects

\begin{tabular}{|c|c|}
\hline & Total subject $(n=91)$ \\
\hline Age (years) & $63.2 \pm 11.6$ \\
\hline Gender (M/F) & $60 / 31$ \\
\hline Body mass index $\left(\mathrm{kg} / \mathrm{m}^{2}\right)$ & $23.6 \pm 3.7$ \\
\hline Waist circumferences (cm) & $84.3 \pm 10.4$ \\
\hline Current smoker (\%) & $34(37.4 \%)$ \\
\hline Current alcohol consumer (\%) & $26(28.6 \%)$ \\
\hline \multicolumn{2}{|l|}{ Daily nutrient intakes } \\
\hline Total energy (kcal) & $2013.9 \pm 579.6$ \\
\hline Carbohydrate (g) & $291.8 \pm 71.2$ \\
\hline$\%$ Carbohydrate (\% for kcal) & $59.2 \pm 8.2$ \\
\hline Protein (g) & $89.1 \pm 32.7$ \\
\hline$\%$ Protein (\% for kcal) & $17.5 \pm 2.4$ \\
\hline Fat (g) & $57.4 \pm 27.2$ \\
\hline$\%$ Fat (\% for kcal) & $24.8 \pm 5.8$ \\
\hline \multicolumn{2}{|l|}{ Vitamin intake } \\
\hline Vitamin A ( $\mu \mathrm{g} R E / d)$ & $1125.4 \pm 633.6$ \\
\hline$\beta$-carotene $(\mathrm{mg} / \mathrm{d})$ & $5.2 \pm 3.4$ \\
\hline Vitamin D $(\mu \mathrm{g} / \mathrm{d})$ & $60.5 \pm 53.9$ \\
\hline Vitamin E (mg $\alpha-T E / d)$ & $17.2 \pm 6.6$ \\
\hline Vitamin C (mg/d) & $167.3 \pm 78.1$ \\
\hline Thiamin (mg/d) & $1.6 \pm 0.6$ \\
\hline Riboflavin (mg/d) & $1.5 \pm 0.5$ \\
\hline Niacin (mg NE/d) & $18.2 \pm 8.3$ \\
\hline Vitamin B6 (mg/d) & $2.6 \pm 0.8$ \\
\hline Folate $(\mu \mathrm{g} / \mathrm{d})$ & $378.5 \pm 126.9$ \\
\hline Vitamin B12 ( $\mu \mathrm{g} / \mathrm{d})$ & $2.6 \pm 2.3$ \\
\hline
\end{tabular}

the morning. Body mass index (BMI) was calculated as body weight in kilograms divided by height in square meters $\left(\mathrm{kg} / \mathrm{m}^{2}\right)$. After overnight fast, venous blood specimens were collected in EDTA-treated tubes $(8 \mathrm{~h}-10 \mathrm{~h})$. The collected samples were kept on ice before being arrived at laboratory. Then, they were centrifuged and separated into plasma and stored at $-70^{\circ} \mathrm{C}$ until analysis of plasma cytokines.

Survey for nutrient intakes. Dietary data were obtained by a nutritionist using a semi-quantitative food-frequency questionnaire (FFQ) of which the validity had been previously tested.(18) The questionnaire was composed of 87 food items commonly used by Koreans. Each subject was asked to report the usual frequency of consumption and usual portion size of the listed items during the past year. Based on this information, nutrient intakes were calculated from Korean food composition tables and other published data. ${ }^{(19-21)}$ The daily nutrient intakes were calculated by multiplying the nutrient content of the specified portion of each food item by the frequency of its daily consumption and summing over all items. Average values of daily intakes of macronutrients and vitamins of study subjects are presented in Table 1 .

Plasma levels of CRP and proinflammatory cytokines. Plasma CRP was measured by an Express Plus autoanalyzer (Chiron Diagnostics Co., MA) using a commercially available high-sensitivity kit, CRP-Latex (II) X2 (Seiken Laboratories Ltd., Tokyo, Japan). Plasma concentrations of IL-1 $\beta$, IL-2, IL-4, IL-6, IL-7, IL-8, IL-10, IL-12, IL-13, IL-17, granulocyte-colony stimulating factor (G-CSF), granulocyte-macrophage (GM)-CSF, interferon (IFN) $-\gamma$, macrophage migration inhibitory factor- $1 \beta$ (MIP-1 $\beta$ ), monocyte chemoattractant protein-1 (MCP-1), and TNF- $\alpha$ were measured using a Bio-Plex human cytokine assay kit (Bio-Rad Laboratories, Inc., Hercules, CA).

Statistical analysis. Statistical tests were performed with SPSS 12.0 software package (Statistical Package for the Social Science, SPSS Ins., Chicago, IL). Each variable was examined for normal distribution and abnormally distributed variables were log-transformed. The data are presented as the mean $\pm \mathrm{SD}$
The Pearson (unadjusted) and partial (adjusted for traditional risk factors for HF) correlation analyses were used to evaluate the relationship among variables. Stepwise multiple regression analysis was used to determine which factor mainly contributes to plasma levels of pro-inflammatory cytokines. $p$ values less than 0.05 were considered statistically significant.

\section{Results}

General characteristics of study subjects. Table 1 presents the mean age $(63.2 \pm 11.6$ years) and body mass index (BMI, $\left.23.6 \pm 3.7 \mathrm{~kg} / \mathrm{m}^{2}\right)$ of the $91 \mathrm{HF}$ patients (60 males and 31 females). Study subjects were in the New York Heart Association (NYHA) functional classes I and II. The mean ejection fraction was $33.9 \pm 10.1 \%$ in the total subjects. Twenty-seven patients had diabetes mellitus and 32 patients had hypertension. The etiology of HF included 56 by ischemic heart disease and 35 by nonischemic heart disease. Medical treatment for all subjects consisted of diuretics $(65.9 \%)$, digitalis $(17.6 \%)$, $\beta$-blockers $(60.4 \%)$, angiotensin converting enzyme (ACE) inhibitors and/or angiotensin II receptor blockers (69.2\%), and hypolipidemic agents $(53.8 \%)$. Table 1 also includes the information of daily nutrient intakes of study subjects.

Correlations of antioxidant vitamin intakes with plasma levels of CRP and inflammatory cytokines. Seventeen kinds of cytokines in plasma were measured in this study. Among these 17 cytokines, those detected in more than $80 \%$ of the total samples were used for further analysis. This included IL-1 $\beta$, IL-5, IL-6, IL-7, IL-8, IL-10, MCP-1, and MIP-1 $\beta$. Correlation analysis showed that dietary intake of vitamin $C, \beta$-carotene and folate among reported vitamins were associated with plasma levels of cytokines as follows. Plasma MCP-1 levels significantly correlated with dietary intakes of vitamin $\mathrm{C}(r=-0.24, p<0.05)$ and folate $(r=-0.31, p<0.001)$. However, the significance maintained only between MCP-1 and dietary folate intake after the adjustment for traditional risk factors for HF (i.e. age, gender, BMI, smoking, alcohol consumption, drug use, and the presence of disease) $(r=$ $-0.28, p<0.01)$. On the other hand, plasma IL-8 levels significantly correlated with dietary intakes of vitamin $\mathrm{C}(r=-0.34$, $p<0.005), \beta$-carotene $(r=-0.38, p<0.001)$, and folate $(r=-0.37$, $p<0.001)$ before and after the adjustment for traditional risk factors (vitamin $\mathrm{C}: r=-0.38, p<0.001, \beta$-carotene: $r=-0.42, p<0.001$, and folate: $r=-0.38, p<0.001)$. In addition, plasma CRP levels significantly correlated with dietary intakes of vitamin $\mathrm{C}(r=-0.30$, $p<0.005)$, $\beta$-carotene $(r=-0.23, p<0.05)$, and folate $(r=-0.31$, $p<0.005)$. However, these significances disappeared after the adjustment.

Stepwise multiple regression analysis to find the major contributors to plasma levels of MCP-1 and IL-8. Stepwise multiple linear regression analysis was used to determine the independent influencing factors on plasma levels of MCP-1 and IL-8. Independent variables included traditional risk factors for HF (i.e. age, gender, BMI, smoking, alcohol consumption, drug use, and the presence of disease), and all the dietary vitamins which showed significant associations as shown in Table 2 . The analysis revealed that dietary folate intake was a primary influencing factor on plasma levels of MCP-1 (adjusted $\beta$-coefficient: $-0.296, p<0.005, \mathrm{R}^{2}=0.20$ ) and IL-8 (adjusted $\beta$-coefficient: $-0.368, p<0.001, \mathrm{R}^{2}=0.32$ ). ACE inhibitor and hypertension were found as secondary factors affecting MCP-1 (adjusted $\beta$ coefficient: $0.213, p<0.05$ ) and IL-8 (adjusted $\beta$-coefficient: -0.291 , $p<0.01$ ), respectively (Table 3 ).

\section{Discussion}

The aim of this study was to examine the relationships between dietary intakes of antioxidant vitamins and the levels of proinflammatory cytokines in Korean HF patients in a cross-sectional 

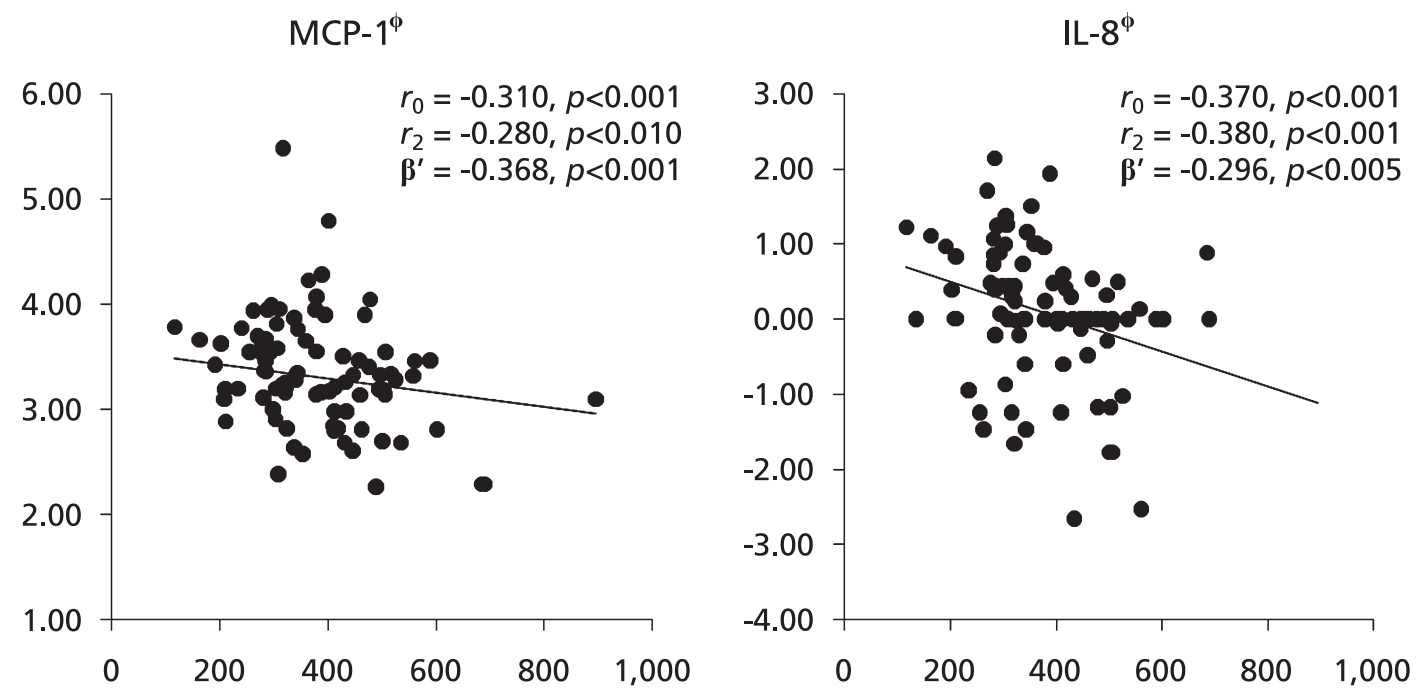

\section{Dietary folate intake $(\mu \mathrm{g} / \mathrm{d})$}

Fig. 1. Relationship between dietary folate intake and plasma levels of MCP-1or IL-8. $\phi$ : log-transformed value, $r$ : Pearson correlation coefficient (unadjusted), $r_{2}$ : Partial correlation coefficient (adjusted for traditional risk factors for heart failure: age, gender, body mass index, smoking, alcohol consumption, drug use, and the presence of diseases). $\beta^{\prime}$ : adjusted $\beta$-coefficient, tested by stepwise multiple regression analysis [Independent variable input: traditional risk factors for $\mathrm{HF}$ including age, gender, body mass index, smoking, alcohol consumption, drug use, and the presence of diseases and dietary antioxidant vitamins intake (vitamin C, $\beta$-carotene and folate)]. MCP-1: monocyte chemoattractant protein-1, IL-8: interleuking-8.

Table 2. Correlations between antioxidant vitamin intakes and plasma pro-inflammatory cytokines in the total HF patients

\begin{tabular}{cllcc}
\hline & & Vitamin C & $\beta$-carotene & Folate \\
\hline C-reactive protein & $r_{0}$ & $-0.30^{* * *}$ & $-0.23^{*}$ & $-0.31^{* * *}$ \\
& $r_{1}$ & $\mathrm{~ns}$ & $\mathrm{~ns}$ & $\mathrm{~ns}$ \\
MCP-1 & $r_{0}$ & $-0.24^{*}$ & $\mathrm{~ns}$ & $-0.31^{* * * *}$ \\
& $r_{1}$ & $\mathrm{~ns}$ & $\mathrm{~ns}$ & $-0.28^{* *}$ \\
IL-8 & $r_{0}$ & $-0.34^{* * *}$ & $-0.38^{* * * *}$ & $-0.37^{* * * *}$ \\
& $r_{1}$ & $-0.38^{* * * *}$ & $-0.42^{* * * *}$ & $-0.38^{* * * *}$
\end{tabular}

Tested by Pearson and partial correlation analysis. $r=$ correlation coefficient. $r_{0}$ : unadjusted, $r_{1}$ : after adjusting for traditional risk factors for HF including age, gender, body mass index, smoking, alcohol consumption, drug use, and the presence of diseases. ${ }^{*} p<0.05,{ }^{* *} p<0.01, * * * p<0.005$, $* * * * p<0.001$. MCP-1: monocyte chemoattractant protein-1, IL-8: interleuking-8.

Table 3. Stepwise multiple regression to identify factors influencing plasma levels of MCP-1 and IL-8

\begin{tabular}{cccccc}
\hline Dependent variable & Independent variable & Adjusted $\beta$-coefficients & $p$ value & $\mathrm{R}^{2}$ & $p$ value \\
\hline \multirow{2}{*}{ MCP-1 } & Dietary folate & -0.296 & $<0.005$ & 0.2 & $<0.001$ \\
& ACEI & 0.213 & $<0.05$ & $<0.001$ & 0.32 \\
IL-8 & Dietary folate & -0.368 & $<0.01$ & $<0.001$
\end{tabular}

Independent variable input: traditional risk factors for HF including age, gender, body mass index, smoking, alcohol consumption, drug use, and the presence of diseases and dietary antioxidant vitamins intake (vitamin C, $\beta$-carotene and folate); MCP-1: monocyte chemoattractant protein-1, IL-8: interleuking-8, ACEl: angiotensin converting enzyme inhibitor, HTN: hypertension.

setting. The results showed that dietary folate intake was found an independent influencing factor on plasma levels of IL-8 and MCP-1.

Several observational and clinical studies have reported that low folate intake is associated with an increased risk of coronary vascular disease and stroke. ${ }^{(22-24)}$ A study conducted in German reported a negative relationship of dietary folate intake with the risk of myocardial infarction which suggested that folate intake may be an important factor for the primary prevention of myocardial infarction. ${ }^{(25)}$ Regarding the association of dietary folate with inflammation, a randomized placebo-controlled study showed that folate supplementation for 12 weeks significantly decreased the levels of IL-8, MCP-1, and CRP in overweight subjects. ${ }^{(26)}$ Furthermore, supplementation of folate and folate plus antioxidants improved endothelial dysfunction estimated with branchial artery flow-mediated dilation in coronary artery disease (CAD) patients, by increasing plasma folate levels. ${ }^{(27)}$ The relationship between folate and pro-inflammatory cytokines is further supported by the data from in vivo and experimental studies. ${ }^{(17,28)}$ Six-month folate treatment in hyperhomocysteinemic patients reduced the amounts 
of MCP-1 and IL-8 secreted by human monocytes. ${ }^{(17)}$ Several experimental and animal studies have also shown that folate inhibits homocysteine-induced NF- $\kappa \mathrm{B}$ activation and MCP-1 expression in macrophages. ${ }^{(28)}$ The exact mechanism for the beneficial effects of folate on inflammation has not been elucidated, but several possible pathways could be involved. One potential mechanism is associated with the ability of folate to reduce homocysteine levels. Hyperhomocysteinemia is a predictive risk marker of $\mathrm{HF}^{(29)}$ and is associated with the severity of HF. ${ }^{(30)}$ Plasma homocysteine levels are strongly influenced by folate, vitamin $\mathrm{B}_{12}$, and $\mathrm{B}_{6}$ and hyperhomocysteinemia might increase the expression of adhesion molecule followed by the immigration and activation of inflammatory cells and the secretion of chemokines such as MCP-1 and IL-8. ${ }^{(31,32)}$ Homocysteine particularly induces the expression and secretion of MCP-1 and IL-8 in human aortic endothelial cells, smooth muscle cell lines and monocyte cell lines via the activation of NF- $\kappa \mathrm{B}$, which results in the increased monocyte chemotaxin. ${ }^{(33-35)} \mathrm{MCP}-1$ and IL- 8 are known to play an important role in the pathogenesis of $\mathrm{HF}$. MCP-1 stimulates the migration of monocytes into the intima of arterial walls and serum IL- 8 is also known as a predictor of HF in patients with myocardial infarction. In fact, IL-8 and MCP-1 are chemokine superfamily as chemotatic factor and have similar structure: mature MCP-1 protein possesses four cysteines at similar position as IL-8. ${ }^{(33)}$ Thus, it is possible that dietary folate reduces MCP-1 and IL- 8 together by decreasing levels of homocysteine which induces secretions of MCP-1 and IL-8 and that's why we could find the significant correlations especially with IL- 8 and MCP-1 in our HF subjects.

Alternatively, folate may have beneficial effects on endothelial function as an antioxidant independent of its lowering of homocysteine. Recently, in vitro evidence has indicated that folate, as a potential antioxidant, can increase nitric oxide (NO) production, directly scavenge superoxide radicals, ${ }^{(36,37)}$ and inhibit microsomal lipid peroxidation. ${ }^{(38)}$ In addition, several studies proposed that 5 methylene tetra-hydrofoalte (5MeTHF), the main circulating metabolite of folate, directly influences endothelial NO synthase (eNOS) activity by stabilizing tetrahydrobiopterin (BH4), a cofactor of the eNOS reaction. ${ }^{(37,39)}$ Therefore, it is possible that folate may suppress pro-inflammatory cytokines such as IL- 8 and MCP-1 through its antioxidant potential and ameliorate endothelial function in HF. In the present study, we observed that dietary folate was an independent influencing factor for the levels of IL- 8 and MCP-1 among many inflammatory markers. Chemokines, including IL-8 and MCP-1, have been implicated in the pathogenesis of type 2 diabetes mellitus and atherosclerosis ${ }^{(40,41)}$ in which they stimulate vascular endothelial cells and the trafficking of macrophages into the arterial intima. Considering that serum IL- 8 was found to be a predictor of HF in patients with myocardial infarction and percutaneous intervention, ${ }^{(42)}$ we can speculate that dietary folate might be associated with the roles of chemokines in immunologic and inflammatory processes in HF. With regard to the adequacy of dietary vitamin intakes in the study subjects, the results showed that about $38 \%$ of the total subjects had lower intakes than the dietary reference intakes (DRIs) but, dietary folate intake met the DRIs for Koreans. This indicates that the proportion of lower intakes of dietary vitamin in HF patients was substantial, which could lead to potential nutritional complications in these patients. Our observations support previous reports indicating that dietary folate intake may be associated with the suppression of inflammatory cytokine levels in HF. Furthermore, it can be suggested that a systemic nutritional education program is especially needed for HF patients as a preventive measure.

Still, however, this issue remains controversial. Several epidemiological and intervention studies have failed to prove a relationship between serum folate level and inflammatory markers. ${ }^{(43,44)}$ It was reported that folate supplementation did not improve inflammation such as IL-6, IL-8, CRP, and MCP-1 despite lowering homocysteine levels. ${ }^{(45,46)}$ Further studies are needed to elucidate the relationship between folate and pro-inflammatory cytokines in various populations, thus explaining the discrepancy in results across studies.

The present study has several limitations. First, we did not measure plasma homocysteine levels, making it difficult to confirm whether the effect of dietary folate on pro-inflammatory markers is related to the reduction of homocysteine. Second, an examination of plasma folate levels is required to clarify folate status in the body as well as dietary folate intake. Third, the cross-sectional study design, small sample size, and use of an FFQ to assess dietary intake are further limitations. Fourth, there was no control group used to compare the levels of dietary vitamin intake and pro-inflammatory markers. Nevertheless, the present study is meaningful because it was conducted in a very homogenous group of Korean subjects with controlled severity of HF.

Despite these limitations, the present study shows the inverse relationship between dietary folate intake and plasma levels of IL- 8 and MCP-1 in HF patients. It may provide a better understanding of the potential anti-inflammatory effects of folate on HF. As evidenced by a recent study showing that serum IL-8 is a predictor of $\mathrm{HF}$, the combined data suggest a major role of folate in the immunologic and inflammatory processes of HF.

\section{Acknowledgment}

This research was supported by a Korea University Grant in 2009.

\section{Abbreviations}

ACE angiotensin converting enzyme

BH4 tetrahydrobiopterin

BMI body mass index

CAD coronary artery disease

CRP C-reactive protein

DRIs dietary reference intakes

FFQ food-frequency questionnaire

G-CSF granulocyte-colony stimulating factor

GM-CSF granulocyte-macrophage colony stimulating factor

HF heart failure

IFN interferon

IL interleukin

LVEF left ventricular ejection fraction

MCP-1 monocyte chemoattractant protein-1

5-MeTHF 5 methylene tetra-hydrofoalte

MIP-1 $\beta$ macrophage migration inhibitory factor- $1 \beta$

NO nitric oxide

NOS NO synthase

NYHA New York Heart Association

TNF- $\alpha$ tumor necrosis factor alpha

\section{References}

1 Gullestad L, Aukrust P. The cytokine network in heart failure: pathogenetic importance and potential therapeutic targets. Heart Fai Monit 2001; 2: 8-13.

2 Aukrust P, Ueland T, Lien E, and et al. Cytokine network in congestive heart failure secondary to ischemic or idiopathic dilated cardiomyopathy. Am J Cardiol 1999; 83: 376-382.

3 Testa M, Yeh M, Lee P, and et al. Circulating levels of cytokines and their endogenous modulators in patients with mild to severe congestive heart failure due to coronary artery disease or hypertension. $\mathrm{J} \mathrm{Am} \mathrm{Col}$. Cardiol 1996; 28: 964-971.

4 Torre-Amione G, Kapadia S, Benedict C, Oral H, Young JB, Mann DL. 
Proinflammatory cytokine levels in patients with depressed left ventricular ejection fraction: a report from the Studies of Left Ventricular Dysfunction (SOLVD). J Am Coll Cardiol 1996; 27: 1201-1206.

5 Kawakami H, Shigematsu Y, Ohtsuka T, and et al. Increased circulating soluble form of Fas in patients with dilated cardiomyopathy. Jpn Circ J 1998; 62: $873-876$.

6 Gullestad L, Aukrust P. Review of trials in chronic heart failure showing broad-spectrum anti-inflammatory approaches. Am J Cardiol 2005; 95: C17C23.

7 Yndestad A, Damås JK, Øie E, Ueland T, Gullestad L, Aukrust P. Role of inflammation in the progression of heart failure. Curr Cardiol Rep 2007; 9: 236-241.

8 Satoh M, Minami Y, Takahashi Y, Nakamura M. Immune modulation: role of the inflammatory cytokine cascade in the failing human heart. Curr Heart Fail Rep 2008; 5: 69-74.

9 Calder PC, Albers R, Antoine JM, and et al. Inflammatory disease processes and interactions with nutrition. Br J Nutr 2009; 101 Suppl: S1-S45.

10 Chen YH, Lin SJ, Chen YL, Liu PL, Chen JW. Anti-inflammatory effects of different drugs/agents with antioxidant property on endothelial expression of adhesion molecules. Cardiovasc Hematol Disord Drug Targets 2006; 6: 279 304.

11 Fischer CP, Hiscock NJ, Penkowa M, and et al. Supplementation with vitamins $\mathrm{C}$ and $\mathrm{E}$ inhibits the release of interleukin-6 from contracting human skeletal muscle. J Physiol 2004; 558 (Pt2): 633-645.

12 Sluijs I, Beulens JWJ, Grobbee DE, van der Schouw YT. Dietary carotenoid intake is associated with lower prevalence of metabolic syndrome in middleaged and elderly men. J Nutr 2009; 139: 987-992.

13 Rao AV, Rao LG. Carotenoids and human health. Pharmacol Res 2007; 55: 207-216.

14 Liu S, Ajani U, Chae C, Hennekens C, Buring JE, Manson JE. Longterm beta-carotene supplementation and risk of type 2 diabetes mellitus: a randomized controlled trial. JAMA 1999; 282: 1073-1075.

15 Wang L, Liu S, Manson JE, Gaziano JM, Buring JE, Sesso HD. The consumption of lycopene and tomato-based food products is not associated with the risk of type 2 diabetes in women. J Nutr 2006; 136: 620-625.

16 Wang G, Dai J, Mao J, Zeng X, Yang X, Wang X. Folic acid reverses hyperresponsiveness of LPS-induced chemokine secretion from monocytes in patients with hyperhomocysteinemia. Atherosclerosis 2005; 179: 395-402.

17 Witte KK, Nikitin NP, Parker AC, and et al. The effect of micronutrient supplementation on quality-of-life and left ventricular function in elderly patients with chronic heart failure. Eur Heart J 2005; 26: 2238-2244.

18 Shim JS, Oh KW, Suh I, and et al. A study on validity of a semiquantitative food frequency questionnaire of Korean adults. Kor J Community Nutr 2002; 7: 484-494.

19 Lee YC, Lee HJ, Oh KW. Fatty acid composition of Korean foods. Seoul: Shin Kwang Publisher, Inc., 1995.

20 Ministry of Health \& Social Welfare, Republic of Korea. Korean food composition table. Seoul: Ministry of Health \& Social Welfare, 1996.

21 National Rural Living Science Institute. Food composition table, 5th Revision., 1996.

22 Rimm EB, Willett WC, Hu FB, and et al. Folate and vitamin B6 from diet and supplements in relation to risk of coronary heart disease among women. JAMA 1998; 279: 359-364.

23 Voutilainen S, Rissanen TH, Virtanen J, Lakka TA, Salonen JT. Low dietary folate intake is associated with an excess incidence of acute coronary events: The Kuopio Ischemic Heart Disease Risk Factor Study. Circulation 2001; 103: 2674-2680.

24 Bazzano LA, He J, Ogden LG, and et al. Dietary intake of folate and risk of stroke in US men and women: NHANES I Epidemiologic Follow-up Study: National Health and Nutrition Examination Survey. Stroke 2002; 33: 11831188.

25 Drogan D, Klipstein-Grobusch K, Dierkes J, Weikert C, Boeing H. Dietary intake of folate equivalents and risk of myocardial infarction in the European Prospective Investigation into Cancer and Nutrition (EPIC)-Potsdam study. Public Health Nutr 2006; 9: 465-471.

26 Solini A, Santini E, Ferrannini E. Effect of short-term folic acid supple- mentation on insulin sensitivity and inflammatory markers in overweight subjects. Int J Obes (Lond) 2006; 30: 1197-1202.

27 Title LM, Cummings PM, Giddens K, Genest JJ Jr., Nassar BA. Effect of folic acid and antioxidant vitamins on endothelial dysfunction in patients with coronary artery disease. J Am Coll Cardiol 2000; 36: 758-765.

28 Au-Yeung KK, Yip JC, Siow YL, O K. Folic acid inhibits homocysteineinduced superoxide anion production and nuclear factor kappa $\mathrm{B}$ activation in macrophages. Can J Physiol Pharmacol 2006; 84: 141-147.

29 Vasan RS, Beiser A, D'Agostino RB, and et al. Plasma homocysteine and risk for congestive heart failure in adults without prior myocardial infarction. JAMA 2003; 289: 1251-1257.

30 Jacques PF, Bostom AG, Wilson PW, Rich S, Rosenberg IH, Selhub J. Determinants of plasma total homocysteine concentration in the Framingham Off-spring cohort. Am J Clin Nutr 2001; 73: 613-621.

31 Kennedy RH, Owings R, Shekhawat N, Joseph J. Acute negative inotropic effects of homocysteine are mediated via the endothelium. Am J Physiol Heart Cir Physiol 2004; 287: H812-H817.

32 Joseph J, Washington A, Joseph L, and et al. Hyperhomocysteinemia leads to adverse cardiac remodeling in hypertensive rats. Am J Physiol Heart Circ Physiol 2002; 283: H2567-H2574.

33 Poddar R, Sivasubramanian N, Dibello PM, Robinson K, Jacobsen DW. Homocysteine induces expression and secretion of monocyte chemoattractant protein-1 and interleukin-8 in human aorticendothelial cells implications for vascular disease. Circulation 2001; 103: 2717-2723.

34 Wang G, Siow YL, O K. Homocysteine induces monocyte chemoattractant protein-1 expression by activating NF- $\mathrm{BB}$ in THP-1 macrophages. Am J Physiol Heart Circ Physiol 2001; 280: H2840-2847.

35 van Aken, Jansen J, van Deventer SJ, Reitsma PH. Elevated levels of homocysteine increase IL-6 production in monocytic Mono Mac 6 cells. Blood Coagul Fibrinolysis 2000; 11: 159-164.

36 Alp NJ, Channon KM. Regulation of endothelial nitric oxide synthase by tetrahydrobiopterin in vascular disease. Arterioscler Thromb Vasc Biol 2004; 24: 413-420.

37 Hyndman ME, Verma S, Rosenfeld RJ, Anderson TJ, Parsons HG. Interaction of 5-methyltetrahydrofolate and tetrahydrobiopterin on endothelial function. Am J Physiol Heart Circ Physiol 2002; 282: H2167-H2172.

38 Joshi R, Adhikari S, Patro BS, Chattopadhyay S, Mukherjee T. Free radical scavenging behavior of folic acid: evidence for possible antioxidant activity. Free Radic Biol Med 2001; 30: 1390-1399.

39 Stroes ES, van Faassen EE, Yo M, and et al. Folic acid reverts dysfunction of endothelial nitric oxide synthase. Circ Res 2000; 86: 1129-1134.

$40 \mathrm{Yu}$ R, Kim CS, Kawada T, and et al. Involvement of leukotactin-1, a novel CC chemokine, in human atherosclerosis. Atherosclerosis $2004 ; 174$ : 35-42.

41 Zozuliñska D, Majchrzak A, Sobieska M, Wiktorowicz K, Wierusz-Wysocka B. Serum interleukin- 8 level is increased in diabetic patients. Diabetologia 1999; 42: 117-118.

42 Dominguez-Rodriguez A, Abreu-Gonzalez P, Garcia-Gonzalez M, Ferrer J. Prognostic value of interleukin- 8 as a predictor of heart failure in patients with myocardial infarction and percutaneous intervention. Int J Cardiol 2006; 111: $158-160$.

43 Friso S, Jacques PF, Wilson PW, Rosenberg IH, Selhub J. Low circulating vitamin $\mathrm{B}(6)$ is associated with elevation of the inflammation marker Creactive protein independently of plasma homocysteine levels. Circulation 2001; 103: 2788-2791.

44 Folsom AR, Desvarieux M, Nieto FJ, Boland LL, Ballantyne CM, Chambless LE. B vitamin status and inflammatory markers. Atherosclerosis 2003; 169: 169-174.

45 Dusitanond P, Eikelboom JW, Hankey GJ, and et al. Homocysteinelowering treatment with folic acid, cobalamin, and pyridoxine does not reduce blood markers of inflammation, endothelial dysfunction, or hypercoagulability in patients with previous transient ischemic attack or stroke: a randomized substudy of the VITATOPS trial. Stroke 2005; 36: 144-146.

46 Peeters AC, van Aken BE, Blom HJ, Reitsma PH, den Heijer M. The effect of homocysteine reduction by B-vitamin supplementation on inflammatory markers. Clin Chem Lab Med 2007; 45: 54-58. 